\title{
Safety of new medicines in young children
}

\section{Imti Choonara}

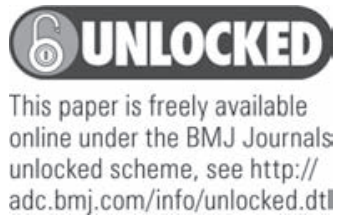

The short report by Tullus (see page 881) highlights safety concerns regarding the use of angiotensin II receptor blockers in preschool children. ${ }^{1}$ Tullus highlights the deaths of three preschool children out of the 183 children with hypertension who received valsartan or candesartan in two clinical trials. One needs to recognise that deaths do occur in clinical trials. A review of paediatric clinical trials published over a 7-year period identified deaths in $11 \%$ of the clinical trials. ${ }^{2}$ In the majority of cases, it was felt by both the authors of the review and the original authors of the published clinical trials that the deaths were unrelated to the investigational drug. There were, however, six clinical trials terminated early because of significant drug toxicity, as well as two trials where the mortality was higher in the treatment group. Fortunately, the risk of death as a direct result of drug toxicity within paediatric clinical trials appears to be low. It is recognised, however, that drug toxicity is poorly reported in clinical trials. ${ }^{3}$ A recent review of over 100 published paediatric clinical trials found that less than one in five of the clinical trials reported safety data adequately. ${ }^{3}$ Clinical trials are not the best way of studying drug toxicity as they are often underpowered to detect severe adverse drug reactions. ${ }^{4}$

Cases of suspected drug toxicity are often reported as individual case reports. Individual case reports may subsequently be confirmed by retrospective reviews or by a greater understanding of the pathophysiology of the toxicity. A good example of the latter is the cardiovascular

Correspondence to Professor Imti Choonara, Academic Division of Child Health, University of Nottingham, Derbyshire Children's Hospital, Derby DE22 3NE, UK; imti.choonara@nottingham.ac.uk collapse of newborn infants following chloramphenicol, which was initially described in three newborn infants in 1959..$^{5}$ Subsequent studies of the metabolism of chloramphenicol in the neonatal period confirmed a considerably decreased capacity to metabolise chloramphenicol and provided a physiological explanation for the toxicity. ${ }^{6}$ Retrospective reviews of all the cases of fatal hepatotoxicity secondary to sodium valproate reported in the USA between 1978 and 1984 identified that the risk of hepatotoxicity was greatest in children under the age of 3 years and those receiving other anticonvulsants alongside the valproate. ${ }^{7}$ The cases of five children who died following the use of a propofol infusion as a sedative in critically ill children were reported in $1992 . .^{8} \mathrm{~A}$ subsequent review of all the reported cases identified that prolonged duration and high doses were more likely to be associated with development of the propofol infusion syndrome (cardiotoxicity, metabolic acidosis and liver damage) in children. ${ }^{9}$ Many other suspected cases of drug toxicity, however, are neither confirmed nor refuted. Concern has previously been expressed that paediatric case reports of individual adverse drug reactions are less likely to be reported than adult cases. ${ }^{10}$

Both the clinical trials of angiotensin II receptor blockers were sponsored by the pharmaceutical industry. This is appropriate as the pharmaceutical company that develops a new product should both sponsor clinical trials and publish the results of the trials. There have unfortunately been several instances where the pharmaceutical company has failed to publish clinical trials when their product has either failed to show efficacy ${ }^{11} 12$ or been associated with significant toxicity. ${ }^{12-14}$ A review of industry sponsored clinical trials of antidepressants identified 74 clinical trials registered with the Food and Drug Administration (FDA). ${ }^{11}$ Thirty-eight of these clinical trials reported a positive benefit of the antidepressant and 37 of these were published. In contrast, 22 of the 36 studies which failed to show efficacy were not published. Additionally,
11 of the 36 studies, which the FDA felt failed to show efficacy, were published with a conclusion suggesting that the drug was effective.

Concern has been expressed by different authors about the interpretation of safety data by the pharmaceutical industry in relation to new products. ${ }^{12-14}$ Rofecoxib was withdrawn in 2004. A meta-analysis of the risk of cardiotoxicity, however, showed that by the end of 2000, data from over 20000 patients had confirmed a highly significant greater risk of myocardial infarction. ${ }^{5}$ The authors felt that rofecoxib should have been withdrawn several years earlier. A review of documents that only became available during legal proceedings against the manufacturer of rofecoxib illustrates how the pharmaceutical company delayed informing the regulatory authorities about an increased number of deaths in the treatment group. ${ }^{16}$ This review also highlights how published articles suggested that the drug was "well tolerated' despite significantly greater deaths in the treatment arm. There have been several new products licensed for use in adults which have had to be withdrawn because of significant drug toxicity. Rimonabant, a treatment for obesity, was withdrawn in 2008 because of its psychiatric toxicity. ${ }^{17}$ In 2009 , the oral $\beta$-agonist orciprenaline, a treatment for asthma, was withdrawn because of cardiotoxicity. ${ }^{18}$ In 2010, rosiglitazone, used for adults with type 2 diabetes, was withdrawn because of the increased cardiovascular risk. ${ }^{19}$ It is fortunately only a minority of medicines that need to be withdrawn because of drug toxicity. However, one always needs to evaluate the risk of toxicity against the advantages of treatment.

History has also shown that young children may be more prone to specific adverse drug reactions than older children. ${ }^{20}$ The retrospective review of cases of hepatotoxicity secondary to sodium valproate identified that young children under the age of 3 years were at the greatest risk. ${ }^{7}$ Antihistamines are widely used in both adults and children. Their use in infants under the age of 1 year, however, has been shown to increase the risk of sleep apnoea, and hence, sudden infant death syndrome. ${ }^{21}$ One therefore needs to be aware that the safety profile of medicines may be different within different paediatric age groups.

New medicines can result in a significant improvement in reducing morbidity 
and mortality. However, one has also to be aware that the safety profile of a new medicine is not established until it has been extensively used for several years. It would seem sensible to use angiotensin II receptor blockers more extensively in older children, and thus establish more safety data, before rushing to use them in preschool children.

Accepted 14 June 2011

Arch Dis Child 2011;96:872-873.

doi:10.1136/archdischild-2011-300450

Competing interests None.

Provenance and peer review Commissioned; internally peer reviewed.

\section{REFERENCES}

1. Tullus K. Safety concerns of angiotensin II receptor blockers in preschool children. Arch Dis Child 2011;Published Online First: 19 June 2011 doi:10.1136/archdischild-2011-300172.

2. Sammons HM, Gray C, Hudson H, et al. Safety in paediatric clinical trials-a 7-year review. Acta Paediatr 2008;97:474-7.

3. de Vries TW, van Roon EN. Low quality of reporting adverse drug reactions in paediatric randomised controlled trials. Arch Dis Child 2009;95:1023-6.
4. Tsang R, Colley L, Lynd LD. Inadequate statistical power to detect clinically significant differences in adverse event rates in randomized controlled trials. J Clin Epidemiol 2009;62:609-16.

5. Sutherland JM. Fatal cardiovascular collapse of infants receiving large amounts of chloramphenicol. Am J Dis Child 1959;97:13-19.

6. Weiss CF, Glazko AJ, Weston JK. Chloramphenico in the newborn infant. A physiologic explanation of its toxicity when given in excessive doses. N Engl J Med 1960;262:787-94.

7. Dreifuss $\mathbf{F E}$, Santilli N, Langer DH, et al. Valproic acid hepatic fatalities: a retrospective review. Neurology 1987:37:379-85.

8. Parke TJ, Stevens JE, Rice AS, et al. Metabolic acidosis and fatal myocardial failure after propofol infusion in children: five case reports. BMJ 1992;305:613-16.

9. Bray RJ. Propofol infusion syndrome in children. Paediatr Anaesth 1998;8:491-9.

10. Impicciatore $\mathbf{P}$, Pandolfini $\mathrm{C}$, Bosetti $\mathrm{C}$, et al Adverse drug reactions in children: a systematic review of published case reports. Paed Perinat Drug Ther 1998;2:27-33.

11. Turner EH, Matthews AM, Linardatos E, et al. Selective publication of antidepressant trials and its influence on apparent efficacy. N Engl J Med 2008;358:252-60.

12. Wieseler B, McGauran N, Kaiser T. Drug studies: a tale of hide and seek. BMJ 2010;341:809-14.

13. Whittington $\mathbf{C J}$, Kendall T, Fonagy $\mathrm{P}$, et al. Selective serotonin reuptake inhibitors in childhood depression: systematic review of published versus unpublished data. Lancet 2004;363:1341-5.

14. Dieppe PA, Ebrahim S, Martin RM, et al. Lessons from the withdrawal of rofecoxib. BMJ 2004;329:867-8.

15. Jüni $\mathbf{P}$, Nartey L, Reichenbach $S$, et al. Risk of cardiovascular events and rofecoxib: cumulative meta-analysis. Lancet 2004;364:2021-9.

16. Psaty BM, Kronmal RA. Reporting mortality findings in trials of rofecoxib for Alzheimer disease or cognitive impairment: a case study based on documents from rofecoxib litigation. JAMA 2008;299:1813-17.

17. Medicines Health Regulatory Agency. Rimonabant: European suspension of marketing authorisation. Drug Safety Update 2008;2:8.

18. Medicines Health Regulatory Agency. Orciprenaline sulphate (Alupent): withdrawal due to unfavourable benefit-risk profile. Drug Safety Update 2009;3:6.

19. Medicines Health Regulatory Agency. Rosiglitazone: recommended withdrawal from clinical use. Drug Safety Update 2010;4:S1

20. Choonara I, Rieder MJ. Drug toxicity and adverse drug reactions in children - a brief historical review. Paed Perinat Drug Ther 2002;5:12-18.

21. Kahn A, Hasaerts D, Blum D. Phenothiazineinduced sleep apneas in normal infants. Pediatrics 1985;75:844-7. 